\title{
GAMIFICATION POR MEIO DE DISPOSITIVOS MÓVEIS NO ENVELHECIMENTO HUMANO
}

\author{
Leo Natan Paschoal, CCHS, UNICRUZ, leonatanpaschoal@gmail.com \\ Myke Morais de Oliveira, CCHS, UNICRUZ, myke.oliveira09@gmail.com \\ Fabrício Soares Kronbauer, CCHS, UNICRUZ, fabriciokronbauer@yahoo.com.br \\ Rafaela Beatriz Coelho de Moura, CCHS, UNICRUZ, rafah_moura@hotmail.com \\ Patricia Mariotto Mozzaquatro, CCHS, UNICRUZ, pmozzaquatro@unicruz.edu.br \\ Solange Beatriz Billig Garces, CCSA, UNICRUZ, sgarces@unicruz.edu.br
}

Resumo. O presente artigo tem como objetivo demonstrar a utilização de técnicas de gamification em dispositivos móveis como ferramentas de apoio ao ensino de informática a um grupo de idosos. O estudo faz parte de um projeto de extensão que possui como objetivo capacitar pessoas com mais de 60 anos na utilização de recursos de sistemas computacionais. Foi desenvolvido um jogo para o sistema operacional android a fim de proporcionar uma melhoria nas habilidades cognitivas, sensoriais e motoras dos participantes do projeto e incentivar a utilização de dispositivos móveis no cotidiano deles.

Palavras-chaves: Jogo Educativo, Terceira Idade, Dispositivos Móveis.

\section{GAMIFICATION BY MOBILE DEVICES IN HUMAN AGING}

Abstract. This article aims to demonstrate the use of the gamification techniques on mobile devices as a support to teaching computer science to a group of older tools. The study is part of an extension project that has aimed to empower people with over 60 years in the resource utilization of computing systems. A game for the android operating system was developed to provide an improvement in cognitive, sensory and motor skills of project participants and encourage the use of mobile devices in everyday them.

Keywords: Game Educational, Elderly, Mobiles.

\section{INTRODUÇÃO}

O ensino vem aderindo aos avanços computacionais e suas tecnologias. Pode-se citar como exemplo a evolução dos Ambientes Virtuais de Aprendizagem (AVAs), que há alguns anos atrás era acessível apenas por computadores desktops, agora pode ser acessado por smartphone, tablets e outros dispositivos móveis (OLIVEIRA E MOZZAQUATRO, 2011). Nos últimos anos, inclusive já existem novas tecnologias aplicadas aos AVAs como computação imersiva (VOSS et. al, 2013) e a computação ubíqua (PIOVESAN, 2011). Outra é a gamification, técnica que será explorada neste estudo.

A gamification é uma técnica da computação que vem sendo aplicada a educação como ferramenta de auxilio nos processos de ensino aprendizagem (ROQUE et.al, 2013). O termo é de origem inglesa, conforme Simões et. al (2012), e não existe tradução adequada para o português, sendo assim neste estudo será utilizado o termo em inglês. Ela possui como objetivo integrar no ensino ferramentas e estratégias utilizadas

V. $12 \mathrm{~N}^{\circ}$ 2, dezembro, 2014 
em jogos, a fim de proporcionar aos alunos novas motivações no aprendizado e aos professores novas metodologias no ensino, tais definições observadas podem ser concluídas pela leitura dos textos de Fardo (2013), Roque; Santos; Geiss (2013) e França; Reategui (2013).

As contribuições tecnológicas para o ensino também devem beneficiar os que poucos têm convivência com ela. Trata-se das pessoas não nativos digitais, ou seja, pessoas que não nasceram na época em que a computação é tão importante em suas vidas, assim, restam aos nativos digitais colaborar e ensinar os benefícios que a informática pode trazer as pessoas. Por meio de projetos de extensão a inclusão digital na terceira idade vem progredindo cada vez mais, isto é possível perceber na escrita de Mozzaquatro et. al (2012) e Paschoal et. al (2014). Os autores Martins e Rolin (2014) afirmam que "é importante considerar que os idosos estão cada vez mais ativos e participativos da sociedade, não podendo ser excluídos dos benefícios trazidos pelo acesso a web, representados pela internet e suas ferramentas”. Neste contexto, este trabalho busca contribuir com os estudos de informática na educação, principalmente quando o foco é a inclusão digital.

Assim, este artigo visa demonstrar a criação de um jogo desenvolvido com o objetivo de testar os conhecimentos de um grupo de idosos no que se refere ao assunto de informática, abordado nas aulas de informática para terceira idade de um projeto de extensão. Para desenvolvimento do jogo foram utilizadas técnicas de gamification como pontuação e nivelamento (ROQUE et. al, 2013) e o mesmo foi desenvolvido para a plataforma android. O objetivo do desenvolvimento deste jogo foi proporcionar uma integração das tecnologias móveis na terceira idade e utilizar recursos de jogos para verificar e reforçar o aprendizado de informática do público.

Salienta-se que o artigo está estruturado da seguinte forma: na seção 2 são descritas considerações sobre a integração de jogos digitais na terceira idade. Na seção 3 é desenvolvido um estudo sobre a utilização e integração de dispositivos móveis na terceira idade. A seção 4 apresenta os paradigmas da gamification. A metodologia utilizada no trabalho é apresentada na seção 5 . Na seção 6 são apresentadas as aplicações desenvolvidas, e a ferramenta Phonegap Build. Os principais resultados obtidos por meio de qualidade do game são apresentados na seção 7. E por fim, na seção 8 são descritas as considerações finais seguidas das referências bibliográficas utilizadas neste estudo.

\section{INTEGRAÇÃO DE JOGOS NO ENVELHECIMENTO}

Com o aumento da população idosa, surgem necessidades em todas as áreas, especialmente da saúde, da assistência social, da educação, do transporte, da cultura e do lazer. E nos tempos de tecnologia, surgem também outras necessidades, como a da inclusão dos idosos.

Nesse sentido, muitos projetos, nessa área, têm sido pensados, especialmente nas universidades que tem programas, como o da Universidade Aberta para Terceira Idade. Esses são projetos de extensão onde os idosos participam de cursos de formação para iniciação ou aprimoramento nas chamadas TICs. Mas, em relação as tecnologias também os jogos virtuais ou videogames tem sido outra possibilidade de participação dos idosos, especialmente como ferramentas de pesquisa e estudo em determinadas áreas, como a saúde, para avaliar capacidades motoras e cognitivas. 
Fazendo um levantamento bibliográfico sobre projetos realizados nessa área de inclusão digital por meio do domínio do uso da internet e de jogos virtuais para a terceira idade pode-se identificar trabalhos como o de Páscoa (2012), que fez uma dissertação de mestrado, na Universidade Técnica de Lisboa, com o objetivo de compreender a contribuição da rede social facebook para a promoção de um envelhecimento ativo. Para isso realizou o estudo com uma turma de informática da Universidade Sênior, da Universidade Técnica de Lisboa, em Portugal. Os idosos entrevistados referiram que as TIC "são um meio de acesso à informação em que a informação circula de modo rápido e acessível, sendo possível atualizar conhecimentos e comunicar ”(p.85).

Marques et al.(2014) apresentaram um trabalho em evento científico na Universidade Federal de Minas Gerais intitulado Jogos Digitais: a tecnologia em prol do resgate da dignidade humana da pessoa idosa. Essa proposta objetivou destacar a inserção social do idoso, o trabalho com raciocínio, memória, atenção e concentração dos idosos, através da descrição de um estudo de caso realizado por meio de um projeto de extensão denominado "Programa de jogos digitais para adultos e idosos", usando serious games (jogos sérios). A conclusão do estudo demonstra de forma concreta de que "é possível fornecer um mecanismo de aprendizagem associado ao entretenimento”(p.1) e que “o projeto tem proporcionado mudanças significativas na vida deste grupo. A associação entre tecnologia , lazer e motivação tem incluído digital e socialmente esses indivíduos”(p.3).

Outro estudo com o uso de jogos virtuais, foi realizado por Mendes (2012) que investigou a aprendizagem de idosos com Doença de Parkinson após treino com 14 sessões de jogos Nintendo Wii Fit Plus ${ }^{\circledR}$, com diferentes demandas cognitivas e motoras. O aprendizado foi a principal medida de resultados. Todavia, concluiu que a capacidade de pacientes com DP em melhorar e reter o desempenho após o treino com os jogos depende da demanda dos jogos, sobretudo a demanda cognitiva e, por isso ressalta a importância da seleção adequada dos jogos para reabilitação.

Também, utilizando o mesmo jogo Nintendo Wii, Alves (2013), fez em seu Trabalho de conclusão de curso uma pesquisa usando o jogo como ferramenta para treinar a funcionalidade e o equilíbrio em idosos, visando melhorar a qualidade de vida dos mesmos. Além disso, avaliaram dados antropométricos. O treinamento com 10 idosos foi realizado em oito sessões, com 60 minutos de duração, realizado duas vezes por semana. A conclusão que a autora chegou foi de que o "Nintendo Wii é uma ferramenta potencial para o programa de treinamento para melhorar a independência funcional dos idosos”(p.2). Outra pesquisa, nessa mesma linha, usando o jogo Nintendo Wii, foi realizada por Lobo (2013), na qual se comparou os efeitos de dois tipos de programas de treinamento de equilíbrio e marcha. Um foi baseado em exercícios convencionais sem a utilização de videogame. Foi, portanto realizado um estudo experimental. Os jogos foram usados no grupo experimental como estímulo cognitivo. Os resultados da pesquisa, conforme relata Lobo (2013, p. 7) “o treino de equilíbrio e marcha associado ao Nintendo Wii Fit ${ }^{\circledR}$, mostrou-se mais eficiente na melhora da eficiência da marcha, das funções cognitivas e da funcionalidade de idosos saudáveis em comparação ao treinamento convencional”. 


\section{UTILIZAÇÃO DE COMPUTAÇÃO MÓVEL NA TERCEIRA IDADE}

Nos tempos atuais, têm-se vivenciado o surgimento de uma sociedade móvel e conectada, com uma variedade de fontes de informação e modos de comunicação disponível (NAISMITH et al., 2004). Para Quinn (2000), m-learning é a interseção de computação móvel e do e-learning por meio de equipamentos computacionais móveis tais como celulares, smartphones, palmtops e notebooks.

Segundo o Instituto Brasileiro de Geografia e Estatística (IBGE, 2010), a população da terceira idade no Brasil cresce rapidamente. A estimativa é que, em 2050, o público com mais de 60 anos represente 60 milhões dos brasileiros. Neste contexto, torna-se necessário desenvolver estratégias para manter esse público atualizado permitindo a aprendizagem contínua (MOL, 2011).

Analisando a situação exposta anteriormente, como o número de pessoas idosas está aumentando a cada dia, Ijsselsteijn et al. (2007) ressaltam a necessidade crescente de oferecer alternativas de entretenimento e aprendizagem a esse público. Assim, uma área atual que encontra-se em estudo chama-se games digitais. Pearce (2008) relata que estudos de jogos digitais com ênfase no público mais velho ainda são raros, pelo fato dessa necessidade ser relativamente nova. Os autores Yee, Duh e Quek (2010) complementam que os jogos em dispositivos móveis para idosos ainda são pouco estudados, este é um desafio promissor.

Mobile game é o termo utilizado para jogo desenvolvido para ser utilizado em dispositivos móveis, que abrangem o universo de celulares, smartphones, tablets e notebooks (MOL, 2011). Neste contexto, ao se pensar em m-learning para pessoas idosas é preciso considerar que o ensino para estas pessoas apresenta especificidades que precisam ser bem pesquisadas e sistematizadas, a fim de desenvolver uma metodologia inclusiva e eficaz.

\section{PARADIGMAS DE TÉCNICAS DE GAMIFICATION}

De acordo com Deterding et.al (2011) e Moreira; Ellwanger (2014) pode-se ter duas dimensões na aplicação de gamification. A primeira consiste em que se podem utilizar elementos e características de um jogo em um contexto que não seja um jogo, e a segunda é que se pode ter um jogo inserido em um contexto (MOREIRA; ELLWANGER, 2014). Para Roque et. al (2013) a gamification é a utilização de técnicas e estratégias que são usadas em jogos em processos que necessariamente não precisam ser jogos e podem ser empregadas em ambientes educacionais.

Lands e Bédard (2011) escrevem que as principais técnicas utilizadas na gamification são de níveis, rankings e barra de progresso. Estas técnicas também são mencionadas por Roque et. al (2013). Já o autor Fardo (2013) afirma que pontos, medalhas e tabelas de lideres são os mecanismos mais básicos de um jogo. Um jogo deve além dos mecanismos mencionados podem ter recompensas, missões, personagens, competição e permitir novas funcionalidades que podem ser adquiridas por pontos. Moreira e Ellwanger (2013) utilizaram em sua aplicação (MobilEduc) as seguintes técnicas: status, configuração de perfil, conquistas, ranking e progresso. Assim, é possível perceber que as pesquisas em gamification vêm utilizando variadas técnicas de jogos no ensino. 


\section{METODOLOGIA}

A fim de esclarecer por meio da literatura científica e experimentos reais, as principais características e dificuldades envolvidas no uso de tecnologias móveis por um publico idoso, o estudo seguiu as seguintes etapas: Definição das ferramentas e técnicas necessárias para a realização do estudo; Instalação e integração das técnicas com as ferramentas; Criação do jogo utilizando técnicas de gamification; Realização de testes de validação; e, Análises e discussão dos resultados relevantes para o estudo.

Para o desenvolvimento do jogo foram desenvolvidas três fases. Estas três fases foram baseadas no estudo de Roque, Santos e Geiss (2013). Sendo elas: desenho, desenvolvimento e testes. A Figura 1 ilustra as atividades de criação do jogo.

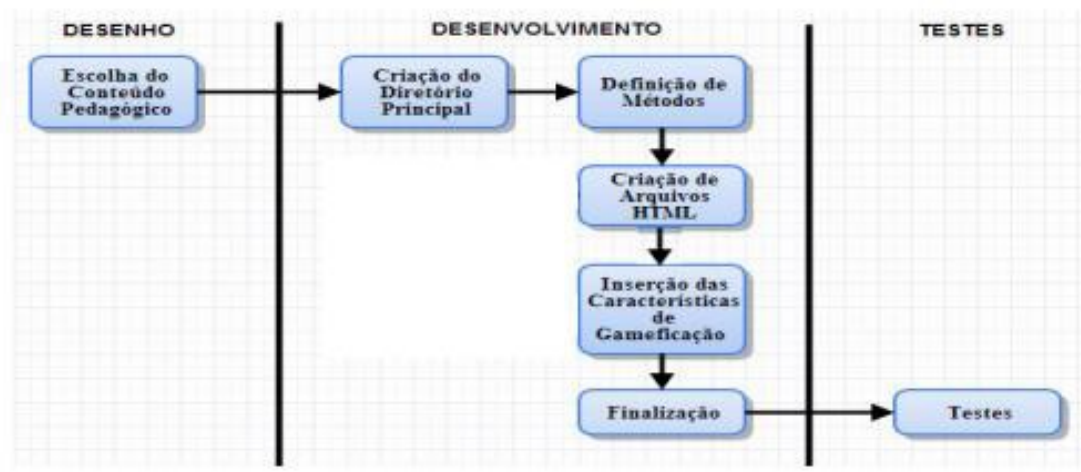

Figura 1. Atividades de criação do Crossword de TI Modelo Adaptado de Roque, Santos e Geiss (2013)

A fase de desenho caracterizou-se pela escolha de conteúdo a ser utilizado no jogo. Na fase de desenvolvimento definiu-se as técnicas de gamification a serem aplicadas no jogo e também a linguagem HTML (HyperText Markup Language), pois o jogo foi todo desenvolvido em HTML e JavaScript e após seu desenvolvimento o mesmo foi convertido em software para ser executado no SO android. Os processos de conversão serão descritos na subseção 6.1. Após a conversão (finalização) realizada na fase de desenvolvimento, inicializou-se a terceira fase (testes). A fase de testes será explicada na seção 7 (Resultados e Discussões).

Nos tópicos seguintes serão apresentados os processos de desenvolvimento do jogo, os resultados obtidos pelo experimento, as considerações para trabalhos futuros a serem desenvolvidos e implementados no jogo e as conclusões finais.

\section{APLICAÇÃO DESENVOLVIDA}

A fim de aplicar os estudos descritos, foi desenvolvido um jogo educativo. Ele recebeu o nome de Crosswords de TI, pois consiste em um jogo de cruzadinhas. Foram inseridas as técnicas de níveis e pontuação por feedback. O mesmo foi desenvolvido especificamente para um grupo de idosos participantes de um projeto de extensão que visa capacitar pessoas com mais de 60 anos na utilização de recursos computacionais.

Foi utilizado a linguagem HTML e JavaScript para criar o jogo, isto pode ser visualizado na Figura 2. Após o desenvolvimento do jogo, o mesmo foi convertido em um aplicativo para o sistema operacional Android, utilizando a ferramenta Phonegap Build. Ainda foram realizados testes para verificar a adaptabilidade do jogo em diferentes dispositivos móveis. Na Figura 3 é possível verificar a tela inicial do jogo, sendo executado no dispositivo Samsung Galaxy Pocket 2 Duos. 


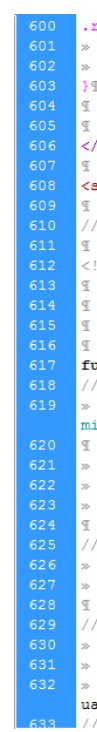

text-align:

Eont-size: 140z; 9

ript type $="$ text $/$ javascript $>$ I

e! icData iq

(1)

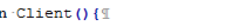

this.min = false; if (document. getElementById) this.

$=$ true; $\}$;

this .ua = navigator. userAgent; 9

this. name $=$ navigator. appName;

this.ver = navigator.appversion;

, ver, indexof ('Mac') $!=-1)$; 9

this, win $=($ this, ver, indexof $($ 'Windows' $), 1=-1), d$

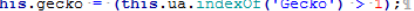

f'(this. gecko) $1 \mathbb{1}$

indexof ('Gecko') +6, this.ua.length))

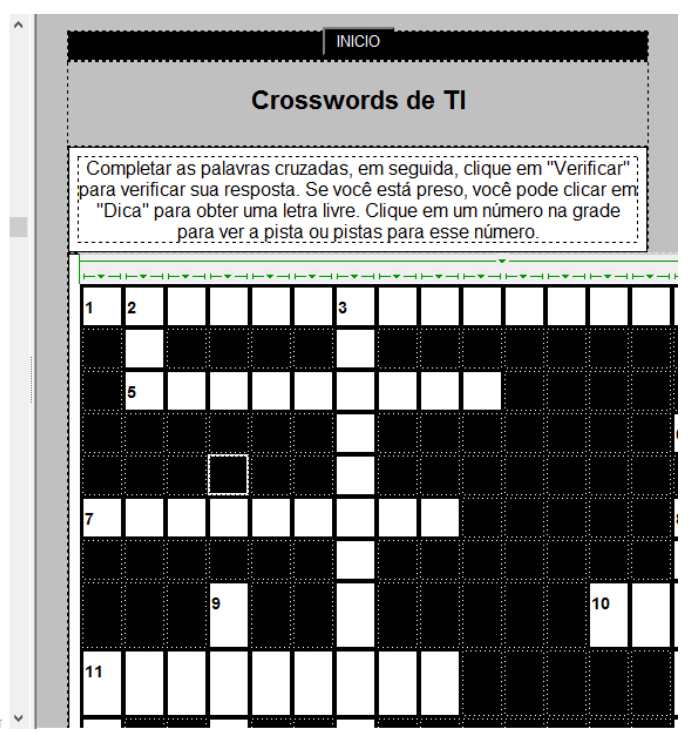

Figura 2. Código do Jogo

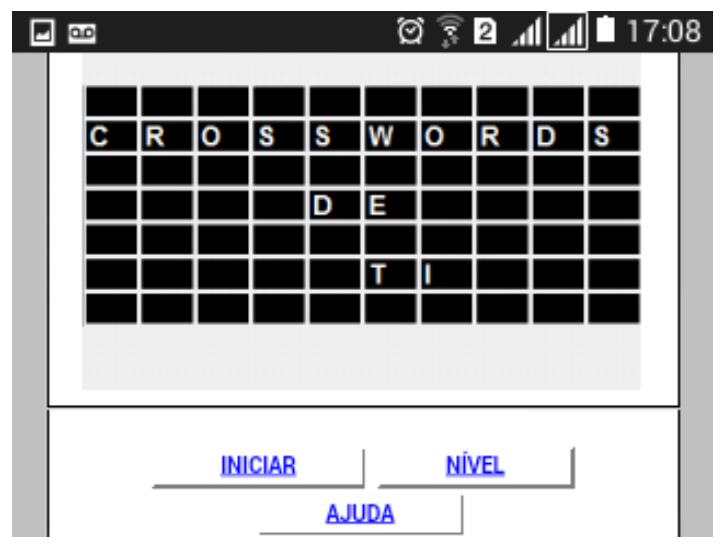

Figura 3. Adaptação no Samsung Galaxy Pocket 2 Duos

Os modelos utilizados para teste e validação foram: Samsung Galaxy Pocket 2 Duos (Android 4.4.2), Samsung Galaxy Young Duos (Android 4.1), Samsung GTS5282 (Android 4.1.2) e Samsung Duos GT- i9300i (Android 4.4.4 kitkat).

\subsection{Phonegap Build}

Neste estudo utilizou-se a ferramenta Phonegap Build, que é um serviço baseado nas nuvens construído em cima do framework Phonegap que permite que o desenvolvedor crie suas aplicações móveis a partir de tecnologias web, sem precisar preparar todo um ambiente de compilação, pois o Build permite compilação diretamente na nuvem (BUILD, 2014). Para fazer uso desta ferramenta, deve-se fazer o upload de um código no formato em HTML. O código é compilado e empacotado e a ferramenta gera um link para download nas seguinte plataformas: andoid, iOS e Windows Phone (BUILD, 2014).

A ferramenta não compila arquivos em outras linguagens como o PHP (Hypertext Preprocessor), por isto utilizou-se nesta pesquisa a linguagem JavaScript. O link para utilizar a ferramenta web pode ser encontrado no endereço: https://build.phonegap.com/apps. 


\section{RESULTADOS E DISCUSSÕES}

A analise dos resultados foi construída, por meio da instalação do jogo em dispositivos móveis dos 11 participantes, com o intuito de observar o comportamento dos participantes em relação ao uso dos dispositivos e do jogo.

A coleta de dados foi feita por meio da análise de questionários que cada participante do projeto preencheu, após o término da validação da aplicação desenvolvida. Para a elaboração do questionário ${ }^{1}$, utilizou-se um método de testes realizados na interface do programa (caixa preta) baseando-se na norma ISO $9126^{2}$ e também foi utilizado um formato para avaliar a satisfação do usuário a produtos, tal formato corresponde à escala de Likert (SANTOS, 2010). Santos (2009) escreve em sua tese que esta escala é um tipo de resposta psicométrica usada frequentemente em séries de questões, "é a escala mais usada em pesquisas de opinião. Ao responderem a um questionário baseado nesta escala, geralmente de 1 a 5 , os perguntados especificam seu nível de concordância com a afirmação” (SANTOS, 2009). O jogo também foi validado, por meio de testes do tipo caixa branca ${ }^{3}$.

A figura 4 apresenta o processo de aprendizagem dos comandos do software, cujo objetivo é verificar se é demorado, assim obteve-se (28\%) de discordância, (27\%) de concordância, (27\%) de indecisão e (18\%) dos participantes discordaram totalmente, sendo que nenhum concordou totalmente $(0 \%)$. O gráfico representado na figura 5 refere-se ao seguinte questionamento: "O aplicativo dispõe de todas as funções necessárias para sua execução?”. Como resposta obteve-se que (91\%) concordaram que o aplicativo dispõe de todas as funcionalidades e (9\%) discordaram totalmente com o questionamento. Nas demais alternativas obteve-se (0\%) como resposta.

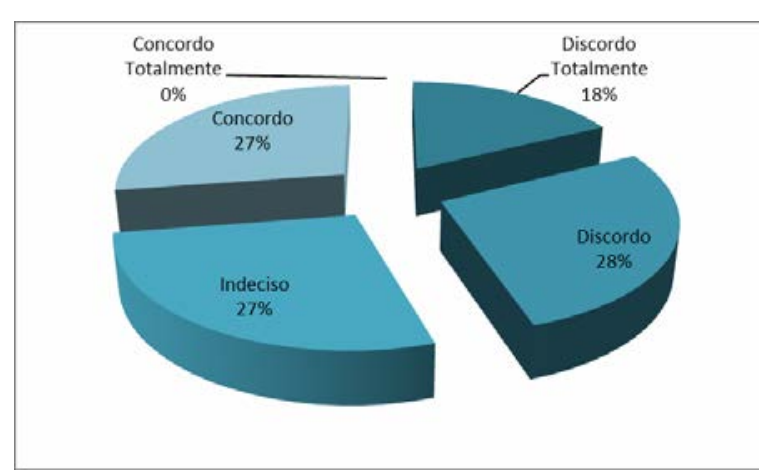

Figura 4. 0 processo de aprendizagem dos comandos do software é demorado?

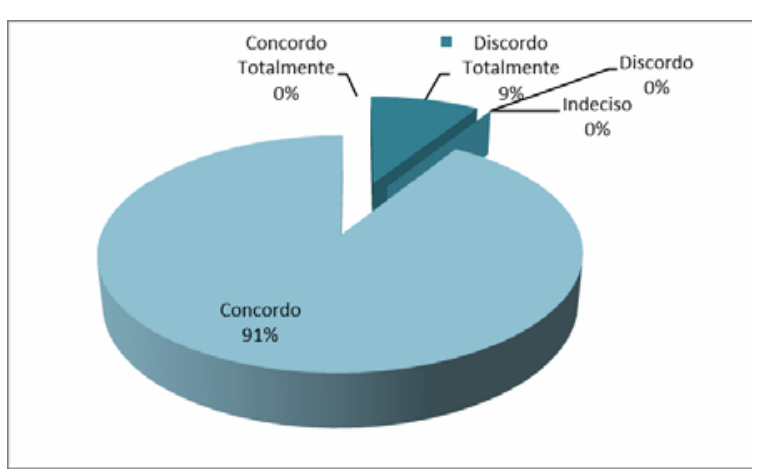

Figura 5. 0 aplicativo dispõe de todas as funções necessárias para sua execução?

Analisou-se também a figura 6. Segundo os respondentes, (55\%) concordou que o tamanho de fonte, imagens e cores facilitam a leitura; (18\%) concordo totalmente com o questionamento, (18\%) discordaram totalmente e $(9 \%)$ ficaram indecisos. O gráfico representado na figura 7 , se refere a indicação, ou seja, se os respondenetes recomendaria eles para outras pessoas. Obteve-se como resposta que 64\% dos participantes concordam, $27 \%$ concordam totalmente e $9 \%$ ficaram indecisos, o que indica que os participantes aprovaram o jogo e inclusive recomendaria o mesmo.

\footnotetext{
${ }^{1}$ O questionário desenvolvido pode ser acessado no seguinte endereço: http://goo.gl/forms/blYxuusrYJ

${ }^{2}$ Norma ISO utilizada para verificar a qualidade de software.

${ }^{3}$ É uma técnica de teste que usa a perspectiva interna do sistema (código fonte). Este teste é realizado pelo desenvolvedor.
} 
Na figura 8, o gráfico referente a seguinte pergunta: “Os titulos dos botões ajudam na navegação?”, obteve-se como resposta de 55\% “concordo totalmente”, 36\% “concordo” e 9\% dos participante marcaram a opção “discordo”. Ao observar a figura 9, percebe que ao questionar o seguinte: "É difícil inicialmente entender como utilizar o aplicativo?", obteve como resposta 46\% “concordo", 36\% “discordo”, 9\% indeciso e $9 \%$ “concordo totalmente”. Com o questionamento representado na figura 09, é possivel perceber que existe uma dificuldade na utilização dos dispositivos móveis. Esta dificuldade pode estar relacionada ao tamanho dos botões, a sensibilidade da tela e também pelos fato dos dispositivos serem touch screen. Assim, deve existir uma preocupação com isto, verificar quais são os problemas e corrigir.

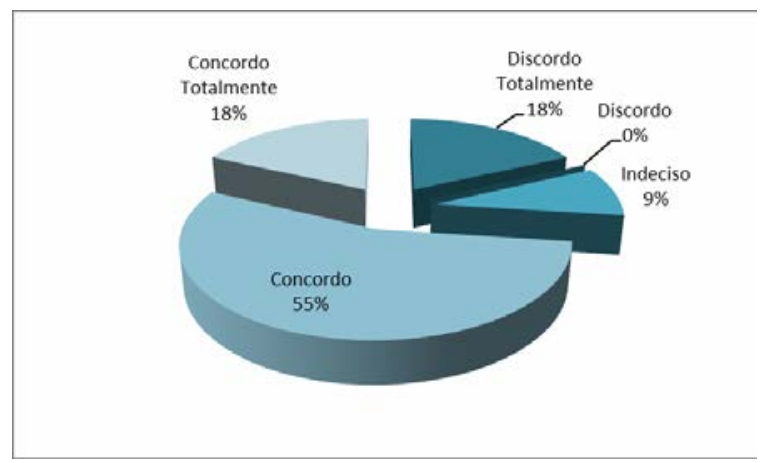

Figura 6. O tamanho de fonte, imagens e cores apresentadas facilitam a leitura?

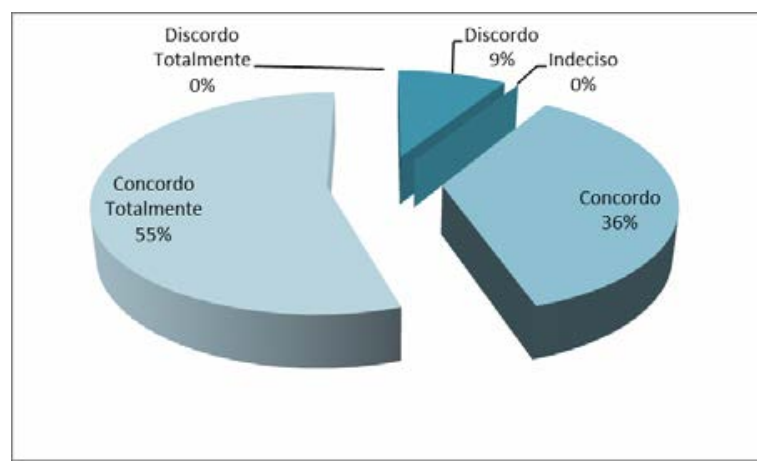

Figura 8. Os títulos dos botões ajudam na navegação?

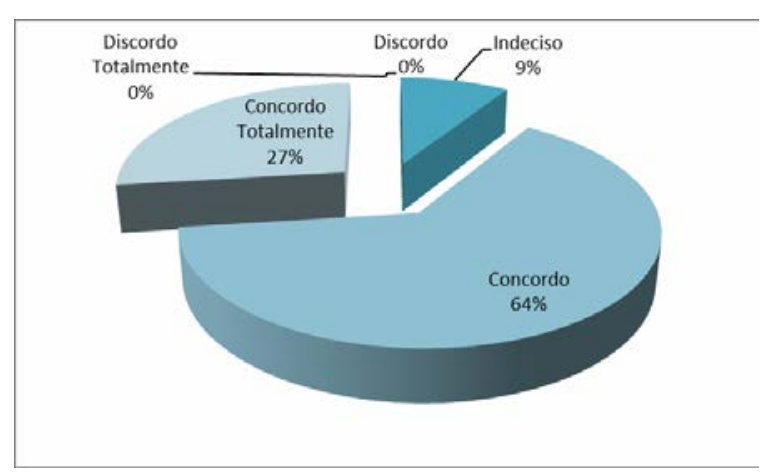

Figura 7. Indicaria o aplicativo para outras pessoas?

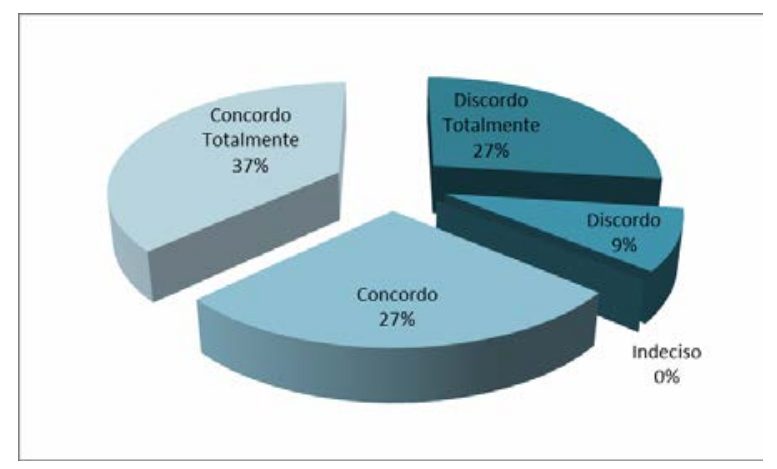

Figura 9. É difícil inicialmente entender como utilizar o aplicativo?

\section{CONSIDERAÇÕES FINAIS}

O artigo proposto objetivou realizar uma aplicação utilizando técnicas de gamification. Para o desenvolvimento da pesquisa foram realizados estudos sobre as técnicas de gamification e sobre a ferramenta Phonegap Build que permite a compilação de arquivos em HTML e JavaScript em arquivos com a extensão para Android, iOS e Windows Phone. Na pesquisa foi utilizada a ferramenta para compilar no formato de extensão “.apk”, pois o objetivo era a utilização em dispositivos móveis com o sistema operacional android.

O jogo desenvolvido possui apenas as técnicas de níveis e feedbacks de pontuação. No entanto, percebe-se que são poucas funções e por isto pretende-se complementar o jogo desenvolvido integrando a ele uma opção de cadastro para jogar online. Nesta nova versão, ele não será especifico para android, por isto será inserido a 
técnica Bootstrap para adaptação em dispositivos móveis, visto que esta técnica permite a adaptação responsiva (VOSS et. al, 2013). Assim, pretende-se inserir as opções ao usuário de criação de personagem, rankings dos melhores jogadores, status de cada personagem, criação de grupos de jogadores. Ainda será modificada a forma de apresentar os níveis do jogo. Em vez de o jogador escolher seu nível, pretende-se disponibilizar para o usuário iniciante apenas o nível básico. Com isso, o jogador deve completar o nível I para jogar o nível II. Com o jogo disponível online existe a possibilidade de integrar a um ambiente de ensino, como o Moodle por exemplo.

\section{REFERÊNCIAS BIBLIOGRÁFICAS}

ALVES, J. C.. Uso do Nintendo Wii como ferramenta de treinamento da funcionalidade, equilíbrio e qualidade de vida em idosos. Muzambinho-MG: Instituto Federal de Educação, Ciência e Tecnologia do Sul de Minas Gerais, 2013. 17 p. Trabalho de Conclusão de Curso.

BUILD. Documentação do PhoneGap Build. Disponível em: <https://build.phonegap.com> Acesso em: Outubro de 2014.

Deterding, S. et al. From game design elements to gamefulness: Defining “gamification”. MindTrek '11 Proceedings of the 15th International Academic MindTrek Conference: Envisioning Future Media Environments. 9-15. (2011).

FARDO, M. L. A gamificação aplicada em ambientes de aprendizagem. RENOTERevista Novas Tecnologias na Educação, v.11, n ${ }^{\circ}$ 1, 2013.

FRANÇA, R. M.; REATEGUI, E. B. SMILE-BR: aplicação de conceitos de gamificação em um ambiente de aprendizagem baseado em questionamento. In. II Congresso Brasileiro de Informática na Educação. Anais, 2013.

IBGE. Síntese de Indicadores Sociais. 2010.

IJSSELSTEIJN, W.A. et al. Digital game design for elderly users. In: PROCEEDINGS OF FUTUREPLAY, 2007, Toronto. Proceedings... Toronto: ACM, 2007. p. 17-22

Lands, N.; Bédard, J. R.(2011). Gamefication Wiki. Disponível em: $<$ http://www.gamification.org $>$.

LOBO, A. M. Efeitos de um treino em ambiente virtual sobre o desempenho da marcha e funções cognitivas em idosos saudáveis. São Paulo: Instituto de Psicologia da Universidade de São Paulo, 2013. 112p. Dissertação de Mestrado.

MARQUES, C. et al. Jogos Digitais: a tecnologia em prol do resgate da dignidade humana da pessoa idosa. Disponível em: <http://icei.pucminas.br/wisi/wpcontent/uploads/2012/08/RobertaNogueira.pdf>. Acessado em 02 nov.2014.

MARTINS, A. D.; ROLIM, A.T. Checklist de uma Abordagem Cognitiva para Avaliar a Acessibilidade das Interfaces Web para Idosos Freqüentadores da UNATI/UEM. In. I Encontro Internacional Tecnologia, Comunicação e Ciência Cognitiva. Anais.

MENDES, F.A.S. Aprendizado motor após treinamento baseado em realidade virtual na Doença de Parkinson: efeitos das demandas motoras e cognitivas dos jogos. São Paulo: Instituto de Psicologia de São Paulo, 2012. 125p. Tese de Doutorado.

MOL, M.A. Recomendações de usabilidade para interface de aplicativos para smartphones com foco na terceira idade. Pontifícia Universidade Católica de Minas Gerais- Belo Horizonte, 2011. Dissertação de Mestrado. 
MOREIRA, G. J.; ELLWANGER, C. Gameficação e padrões de interface para dispositivos móveis no estabelecimento do processo de ensino e de aprendizagem. In: $\mathbf{3}^{\mathbf{o}}$ Seminário Nacional de Inclusão Digital. Anais, 2014.

MOZZAQUATRO, P. M.; et al. Capacitando a terceira idade na utilização de ferramentas tecnológicas. In: XVI Seminário Interinstitucional de Ensino, Pesquisa e Extensão. Anais, 2012.

NAISMITH, L.; LONSDALE, P.; VAVOULA, G.; Shet Sharples, M. S. REPORT 11: Literature Review in Mobile Technologies and Learning. NESTA FUTURELAB SERIES, University of Birmingham. 2004

OLIVEIRA, L.C ; MOZZAQUATRO, P. M. Estudo sobre cloud computing: um novo paradigma para e-learning e m-learning. In: XV Seminário interinstitucional de Ensino, Pesquisa e Extensão. Anais, 2011.

PASCHOAL, L. N.; et.al Software Educacional para o Ensino do Paint para pessoas com mais de 60 anos. In: $3^{\circ}$ Seminário Nacional de Inclusão Digital. Anais, 2014.

PÁSCOA, G.M. G. O contributo da web social - rede social Facebook - para a promoção do envelhecimento ativo: estudo de caso realizado na USALBI. Instituto Superior de Ciências Sociais e Políticas da Universidade Técnica de Lisboa. Lisboa-PT: UTL, 2012. 262p. Dissertação de Mestrado.

PEARCE, C. The truth about baby boomer gamers: A study of over-forty computer game players. Games and Culture, n.3, 2008. p.142-174.

PIOVESAN, S.D. U-SEA: Um Ambiente de Aprendizagem Ubíquo utilizado cloud computing. Universidade Federal de Santa Maria, 2011. 84p. Dissertação de Mestrado.

QUINN, C. mLearning: Mobile, Wireless, In-Your-Pocket Learning. 2000. Disponível em: http://www.linezine.com/2.1/features/cqmmwiyp.htm. Acesso em: nov 2014.

ROQUE, A.S.; et al. Técnicas de Gameficação em AVAs: Um Estudo de Caso no Ambiente Virtual de Aprendizagem Moodle. In: IV Encontro Anual de Tecnologia da Informação. Anais, 2013.

ROQUE, A.S.; SANTOS, C.P.; GEISS, E.R. GameLearning e suas Contribuições ao Ambiente Virtual de Aprendizagem Moodle. In XVIII Conferência Internacional sobre Informática na Educação• TISE. Anais, 2013.

SANTOS, L.M.A. A inserção de um agente conversacional animado em um ambiente virtual de aprendizagem a partir da teoria da carga cognitiva. Universidade Federal do Rio Grande do Sul, 2009. Tese de Doutorado.

SIMÕES, J.; AGUIAR, A.; REDONDO, R.; VILAS, A. Aplicação de elementos de jogos numa plataforma de aprendizagem social. In: II Congresso Internacional TIC e Educação, Portugal. Anais 2012.

VOSS, G.B.; et. al Ambientes Virtuais de Aprendizagem e Ambientes Imersivos: um estudo de caso utilizando tecnologias de computação móvel. In: Simpósio Brasileiro de Informática na Educação, 2013. Anais.

YEE, Y. C.L.S; DUH, B.L.H; QUEK, F. Investigating narrative in mobile games for seniors. In: International Conference on Human Factors in Computing Systems, 28, 2010, New York. Proceedings... New York: ACM, 2010. 